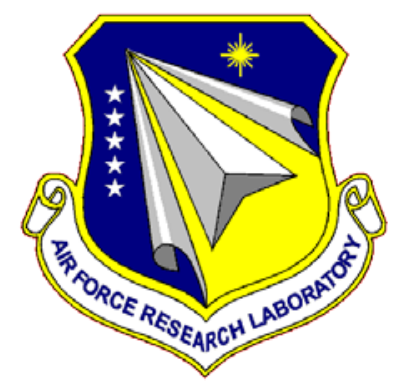

\title{
AFRL-RY-WP-TR-2016-0006
}

\section{SENSITIVITY SIMULATION OF COMPRESSED \\ SENSING BASED ELECTRONIC WARFARE RECEIVER USING ORTHOGONAL MATCHING PURSUIT ALGORITHM}

Lihyeh Liou and David Lin

RF/EO Subsystems Branch

Aerospace Components \& Subsystems Division

Ethan Lin and Chien-In Chen

Wright State University

FEBRUARY 2016

Interim Report

Approved for public release; distribution unlimited.

See additional restrictions described on inside pages

STINFO COPY

AIR FORCE RESEARCH LABORATORY SENSORS DIRECTORATE

WRIGHT-PATTERSON AIR FORCE BASE, OH 45433-7320

AIR FORCE MATERIEL COMMAND

UNITED STATES AIR FORCE 


\section{NOTICE AND SIGNATURE PAGE}

Using Government drawings, specifications, or other data included in this document for any purpose other than Government procurement does not in any way obligate the U.S. Government. The fact that the Government formulated or supplied the drawings, specifications, or other data does not license the holder or any other person or corporation; or convey any rights or permission to manufacture, use, or sell any patented invention that may relate to them.

This report was cleared for public release by the USAF 88th Air Base Wing (88 ABW) Public Affairs Office (PAO) and is available to the general public, including foreign nationals. Copies may be obtained from the Defense Technical Information Center (DTIC) (http://www.dtic.mil).

AFRL-RY-WP-TR-2016-0006 HAS BEEN REVIEWED AND IS APPROVED FOR PUBLICATION IN ACCORDANCE WITH ASSIGNED DISTRIBUTION STATEMENT.

// Signature//

LIHYEH L. LIOU

Program Manager

RF/EO Subsystems Branch

Aerospace Components \& Subsystems Division
// Signature//

JASON G. CROSBY, Major, USAF

Acting Chief

RF/EO Subsystems Branch

This report is published in the interest of scientific and technical information exchange, and its publication does not constitute the Government's approval or disapproval of its ideas or findings.

*Disseminated copies will show “//Signature//” stamped or typed above the signature blocks. 


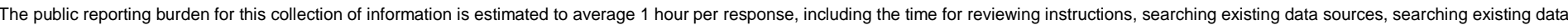

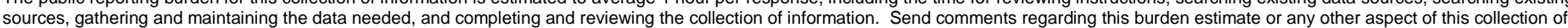

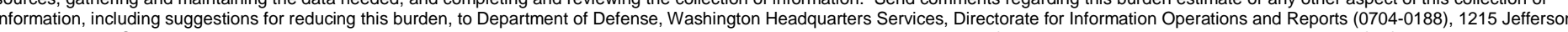

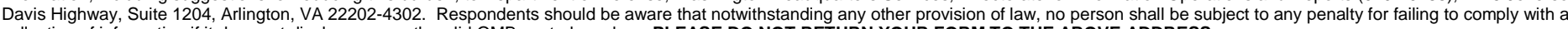
collection of information if it does not display a currently valid OMB control number. PLEASE DO NOT RETURN YOUR FORM TO THE ABOVE ADDRESS.

1. REPORT DATE (DD-MM-YY)

2. REPORT TYPE

3. DATES COVERED (From - To)

February 2016

Interim

8 January 2014 - 31 July 2014

4. TITLE AND SUBTITLE

SENSITIVITY SIMULATION OF COMPRESSED SENSING BASED

ELECTRONIC WARFARE RECEIVER USING ORTHOGONAL MATCHING PURSUIT ALGORITHM

5a. CONTRACT NUMBER

In-house

5b. GRANT NUMBER

5c. PROGRAM ELEMENT NUMBER $62204 \mathrm{~F}$

6. $\operatorname{AUTHOR}(\mathrm{S})$

Lihyeh Liou and David Lin (AFRL/RYDR)

Ethan Lin and Chien-In Chen (Wright State University)

5d. PROJECT NUMBER

2002

5e. TASK NUMBER

$\mathrm{N} / \mathrm{A}$

5f. WORK UNIT NUMBER

YOVS

7. PERFORMING ORGANIZATION NAME(S) AND ADDRESS(ES)

8. PERFORMING ORGANIZATION REPORT NUMBER

Air Force Research Laboratory

Sensors Directorate (AFRL/RYDR)

Wright-Patterson Air Force Base, OH 45433-7320

Air Force Materiel Command

United States Air Force

\section{SPONSORING/MONITORING AGENCY NAME(S) AND ADDRESS(ES)}

Air Force Research Laboratory

Sensors Directorate

Wright-Patterson Air Force Base, OH 45433-7320

Air Force Materiel Command

United States Air Force
Wright State University

Department of Electrical

Engineering

3640 Colonel Glenn Hwy

Dayton, $\mathrm{OH} 45435$

2. DISTRIBUTION/AVAILABILITY STATEMENT

Approved for public release; distribution unlimited.

13. SUPPLEMENTARY NOTES

The U.S. Government is joint author of the work and has the right to use, modify, reproduce, release, perform, display or disclose the work. PAO case number 88ABW-2014-2752, Clearance Date 13 August 2014. Report contains color.

14. ABSTRACT

The wideband coverage of the traditional fast Fourier transform (FFT)-based electronic warfare (EW) receiver is limited by the sampling frequency of the analog-to-digital converter (ADC). Compressed sensing (CS) based wideband EW digital receiver has been proposed and under development to process wideband sparse signals with reduced sampling rate. In this study, we calculated the sensitivity of a CS-based EW receiver. Two modulation schemes of CS are used. One is pseudorandom chip (PRC) and the other is non-uniform sampling (NUS) modulation. The orthogonal matching pursuit (OMP) algorithm is used to process CS data. The insufficient nature of the sparcity of the signal adversely affects the signal detection probability for both PRC and NUS modulation schemes. Methods to remedy this problem in both modulation schemes are proposed. The improvement in the signal detection and thus the sensitivity performance of the CS based receiver are presented.

15. SUBJECT TERMS

electronic warfare, radio frequency, signal detection, signal processing

16. SECURITY CLASSIFICATION OF:

a. REPORT

b. ABSTRACT

c. THIS PAGE

Unclassified
17. LIMITATION OF ABSTRACT:

SAR
NUMBER OF PAGES

18 19a. NAME OF RESPONSIBLE PERSON (Monitor) Lihyeh Liou

19b. TELEPHONE NUMBER (Include Area Code) $\mathrm{N} / \mathrm{A}$ 


\section{Table of Contents}

Section

Page

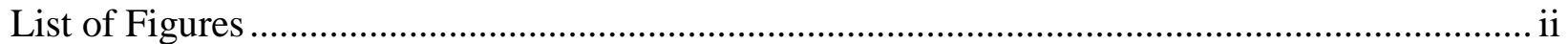

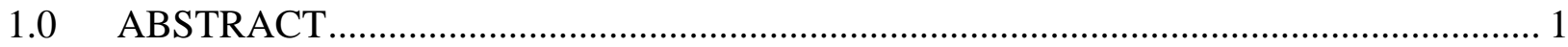

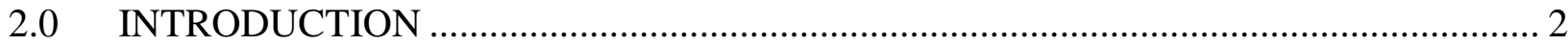

2.1 CS-Based Receiver Sensitivity Simulation Using OMP .............................................. 3

2.1.1 Routine of Receiver Sensitivity Simulation.......................................................... 3

2.1.2 Pseudo-Random-Chip and Non-Uniform Measurement Matrix: ............................ 3

2.1.3 Orthogonal Matching Pursuit........................................................................... 4

2.2 Results of CS-Based Receiver Sensitivity Simulation Using OMP............................. 5

2.2.1 PRC Results and Discussion ....................................................................... 5

2.2.2 NUS Results and Discussion ..................................................................... 7

3.0 SUMMARY AND FUTURE WORK ...................................................................... 10

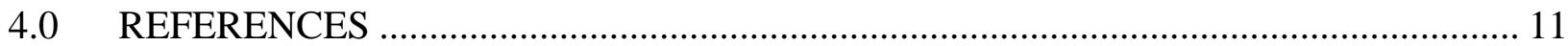

LIST OF SYMBOLS, ABBREVIATIONS, AND ACRONYMS …........................................ 12 


\section{List of Figures}

Figure

Page

Figure 1: OMP Algorithm.......................................................................................... 4

Figure 2: Probability Density Function of PRC CS Scheme Receiver ...................................... 5

Figure 3: Detection Probability vs. S/N for PRC Case without Remedy ................................... 6

Figure 4: Detection Probability vs. S/N for PRC Case with Remedy ........................................ 6

Figure 5: Probability Density Function of NUS CS Scheme Receiver .................................... 7

Figure 6: Detection Probability vs. S/N for NUS Case without Remedy .................................. 7

Figure 7: Probability of Detection vs. S/N when the Input Frequency is restricted to at

Quarter, Half, and Zero Integer away from the Fourier Bins ................................................. 8

Figure 8: Detection Probability vs. S/N for NUS Case with Remedy ...................................... 9

Approved for public release; distribution unlimited. 


\subsection{ABSTRACT}

The wideband coverage of the traditional Fast Fourier transform (FFT)-based electronic warfare $(\mathrm{EW})$ receiver is limited by the sampling frequency of the analog-to-digital converter (ADC). Compressed sensing (CS) based wideband EW digital receiver has been proposed and under development to process wideband sparse signals with reduced sampling rate. In this study, we calculate the sensitivity of a CS based EW receiver. Two modulation schemes of CS are used. One is pseudorandom chip (PRC) and the other is non-uniform sampling (NUS) modulation. The orthogonal matching pursuit (OMP) algorithm is used to process CS data. The insufficient nature of the sparcity of the signal adversely affects the signal detection probability for both PRC and NUS modulation schemes. Methods to remedy this problem in both modulation schemes are proposed. The improvement in the signal detection and thus the sensitivity performance of the CS based receiver are presented. 


\subsection{INTRODUCTION}

Electronic Intelligence Receiver (ELINT) is an important component in EW and layer sensing. The information provided by its function of constant surveillance can be used to detect, track and classify signals across the electromagnetic spectrum. The proper identification and reaction to the threat can avoid disaster and assure spectrum dominance for Air Force systems. To meet the challenges in today's and tomorrow's EW environment where signals are increasingly sophisticated, more dynamic and more crowded in radio frequency (RF) spectrum compared to yesterday, digital wideband receiver is under intensive development. The current state-of the-art for digital receiver bandwidth coverage is now reaching multi-GHz. The conventional wideband digital ELINT receiver design is based on the Nyquist information theory, whereby, bandwidth coverage is limited by the Nyquist sampling rate. Performance therefore, depends on the current high speed ADC technology and computation hardware such as field-programmable gate array (FPGA). While these technologies are advancing, the progress of the improvement may not keep up with the demand of ELINT requirements driven by next generation cognitive and broadband EW capability needs. Innovative designs are proposed to keep abreast with the requirement while to stay within the current technology limitation. One potential leap-ahead is to apply compressed sensing concept for ELINT receiver development.

CS technique provides an alternate solution. It has received significant research attention since the fundamental theory was proved by E. Candès, J. Romberg, and T. Tao in 2006 [1]. In this paper, the authors indicated that the band limited by the Nyquist sampling rate is too restrictive. It is particularly true when the signal is sparse, i.e., the signal can be represented by only a few significant components in an orthogonal basis. Under the sparse condition, one can devise a sampling scheme that takes a much reduced data rate compared to that of Nyquist sampling rate and still cover the same bandwidth as the Nyquist sampling case does. The sampling scheme usually involves modulating the incoming signal and sampling a data after summing the modulated signal over an interval of several chips. The Nyquist waveform can be reconstructed using the reduced sampling if the measurement matrix representing the sampling scheme has the restrictive isometric property (RIP).

In this study, we include two measurement matrices which both exhibit high probability of RIP. One is the PRC, and the other is NUS. The PRC's measurement matrix element is a pseudorandom bit of +1 and -1 with equal probability. The scheme was proposed [2] for image processing using single pixel camera, where the field of view was masked by a grid structure of random +1 and -1 pattern. The NUS scheme was proposed and fabricated by Defense Advanced Research Projects Agency's (DARPA) analog-to-information (A-to-I) program initiated in 2008 [3]. In this scheme, an internal Nyquist rate (i.e., chip rate of modulation) of $4.8 \mathrm{GHz}$ is applied. The sampling time is a pseudorandom integer number within a certain range of time grid determined by the internal Nyquist rate. The average sampling rate is about $300 \mathrm{MHz}$ and the average down sampling rate is about 16.

Many reconstruction algorithms have been proposed to restore the original Nyquist waveform of sparse signal from the reduced sampling data set. These reconstruction algorithms have a range of complexity and accuracy. We apply OMP to assess the sensitivity of the receiver. OMP is a restoration algorithm developed at an early stage [4]. The algorithm is to locate and calculate the

Approved for public release; distribution unlimited. 
signal's component iteratively. There are two major computation steps in every iteration. One is to correlate the residual measurement data with the measurement basis to locate and update the signal component. The other is to update signal's components using least square fit scheme. The computation, compared to the later developed algorithms including NESTA [5], is less complex and yet it has yielded reasonably accurate frequency results.

\subsection{CS-Based Receiver Sensitivity Simulation Using OMP}

\subsubsection{Routine of Receiver Sensitivity Simulation}

Receiver's sensitivity calculation deals only one signal, while dynamic range (DR) deals two or more signals [6]. The DR simulation of CS-based receiver will be conducted in future study.

There are two stages in the routine of sensitivity simulation. The first is to determine the threshold of the detection which depends on the false alarm rate [6]. In this stage, one needs to build an ensemble to set up probability density function (PDF) of the stochastic parameter of the observed parameter at the output port when a Gaussian white noise is at the input port. Result from this stage is the detection threshold to be used in the second stage. The second stage calculates the detection probability $\left(\mathrm{P}_{\mathrm{d}}\right)$ vs. signal-to-noise $(\mathrm{S} / \mathrm{N})$ ratio. The default false alarm rate is $10^{-7}$ in this study. At each $S / N$ point, $P_{d}$ is calculated from the average of 10,000 runs. Sensitivity is defined at the $\mathrm{S} / \mathrm{N}$ (in $\mathrm{dB}$ ) when probability of detection is at $90 \%$.

\subsubsection{Pseudo-Random-Chip and Non-Uniform Measurement Matrix:}

A schematic CS representation is given by:

$$
y_{M \times 1}=\Phi_{M \times N^{x} N \times 1}=\Phi_{M \times N^{\Psi}}{ }_{N \times N^{s} N \times 1}
$$

where $y$ and $\mathrm{x}$ are the measurement and Nyquist vectors, respectively. The $\Phi$ is the measurement matrix with a dimension of $\mathrm{M}$ by $\mathrm{N}$. The $\Psi$ is the basis matrix. The Fourier basis is used in this study. The $\mathrm{s}$ is a sparse vector in frequency domain.

A schematic of $\Phi$ using PRC modulation is shown as:

$$
\Phi=\left[\begin{array}{cccccccccccc}
1 & -1 & 1 & 1 & -1 & -1 & 1 & 1 & -1 & -1 & 1 & -1 \\
-1 & -1 & -1 & 1 & 1 & -1 & 1 & -1 & 1 & 1 & 1 & -1 \\
-1 & 1 & -1 & -1 & 1 & 1 & -1 & 1 & 1 & -1 & -1 & 1 \\
1 & 1 & 1 & -1 & -1 & 1 & -1 & 1 & 1 & -1 & 1 & -1 \\
. & . & . & . & . & . & . & . & . & . & . & . \\
. & . & . & . & . & . & . & . & . & . & . & . \\
1 & 1 & -1 & 1 & -1 & 1 & 1 & -1 & -1 & 1 & 1 & -1 \\
-1 & -1 & 1 & -1 & -1 & -1 & 1 & 1 & -1 & 1 & -1 & 1
\end{array}\right]
$$

where each entry in the matrix is either +1 or -1 with an equal probability among them. In this study, the dimension of the measurement matrix is 36x256, and thus the sampling reduction rate is 7.1. 
A schematic of $\Phi$ using NUS modulation is shown as:

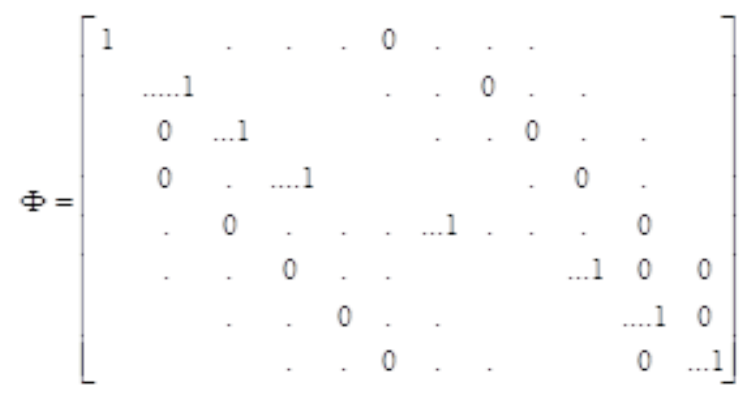

where each row has only one non-zero entry of 1 and the rest elements are zero. The distance between the adjacent row's non-zero entries is a random integer number within a rage. In this study, it is between 7 and 26 with a uniform distribution. The dimension of the NUS measurement matrix is $36 \times 645$ in this study, and thus the sampling reduction rate is 17.9.

\subsubsection{Orthogonal Matching Pursuit}

The OMP algorithm is depicted in Figure 1 [5]:

$$
\begin{array}{ll}
\text { 1: } & \mathbf{r}^{(0)} \leftarrow \mathbf{y} \\
\text { 2: } & \Lambda^{(0)} \leftarrow \varnothing \\
\text { 3: } & \text { for } i=1, \ldots, k \text { do } \\
\text { 4: } \quad \lambda^{(i)} \leftarrow \arg \max _{j=1, \ldots, n}\left|\left\langle\mathbf{r}^{(i-1)}, \phi_{j}\right\rangle\right| \\
\text { 5: } \quad \Lambda^{(i)} \leftarrow \Lambda^{(i-1)} \cup \lambda^{(i)} \\
\text { 6: } \quad \Phi^{(i)} \leftarrow\left[\Phi^{(i-1)} \phi_{\lambda^{(i)}}\right] \\
\text { 7: } \quad \mathbf{x}^{(i)} \leftarrow \arg \min _{\hat{\mathbf{x}}}\left\|\mathbf{y}-\Phi^{(i)} \hat{\mathbf{x}}\right\|_{2} \\
\text { 8: } \quad \mathbf{a}^{(i)} \leftarrow \Phi^{(i)} \mathbf{x}^{(i)} \\
\text { 9: } \quad \mathbf{r}^{(i)} \leftarrow \mathbf{y}-\mathbf{a}^{(i)} \\
\text { 10: } \\
\text { 11: } \hat{\mathbf{x}} \leftarrow \mathbf{x}^{(k)} \\
\text { 12: } \text { return } \hat{\mathbf{x}}, \Lambda^{(k)}, \mathbf{a}^{(k)}, \mathbf{r}^{(k)}
\end{array}
$$

\section{Figure 1: OMP Algorithm}

where steps 4 and 7 are most computation heavy. Step 4 is to find signal's component through correlating the residuals of the measurement vector with each column of the measurement matrix. Step 7 is to calculate the updated Nyquist vector through least square method. Since the incoming signal is presented using real number and the Fourier basis matrix is a complex matrix, there are two components in Fourier bases for each frequency components in the incoming signal.

Approved for public release; distribution unlimited. 


\subsection{Results of CS-Based Receiver Sensitivity Simulation Using OMP}

\subsubsection{PRC Results and Discussion}

Figure 2 shows the PDF of the PRC CS-based Receiver using OMP. The PDF can be approximated with a two half Gaussians and a fat tail (i.e., exponential decade) as in the following equation:

$$
\begin{aligned}
& p(x)=16898 \exp \left[\frac{-(x-0.3161)^{2}}{0.004706}\right] ; 0.3161 \geq \mathrm{x} \\
& 3 \quad p(x)=16898 \exp \left[\frac{-(x-0.3161)^{2}}{0.03302}\right] ; 0.5233 \geq \mathrm{x}>0.3161 \\
& p(x)=4636 \exp [-20.82(x-0.5233)] ; \mathrm{x}>0.5233
\end{aligned}
$$

where $\mathrm{x}$ is the random number of the output of the PRC-based receiver using OMP when Gaussian noise is at the input. The threshold is 1.159 when false alarm rate is $10^{-7}$.

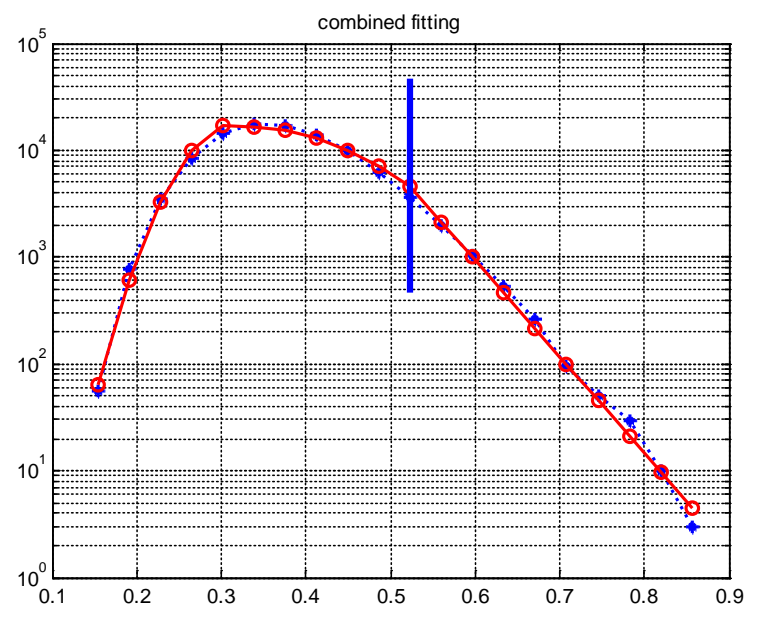

Figure 2: Probability Density Function of PRC CS Scheme Receiver

Figure 3 shows the detection probability vs. S/N. There are two curves in this figure. One is the result according to the detection threshold, and the other is the detection according to the accuracy of the frequency determination. For the latter, the detection is claimed if the determined frequency is accurate within one Fourier frequency bin (frequency resolution in FFT of the frame-sized data points). One notes that the frequency-determined detection only reach to about $96 \%$ when $\mathrm{S} / \mathrm{N}$ increases. Its significance is that there is about $4 \%$ that frequency is wrongly determined even signal is claimed according to threshold criterion. This nuisance deserves attention. A close look into cases giving wrong frequency results found the problem is due to sparcity. This is evidenced when the input frequency is not at the Fourier bins, and the signal power spreads over to the neighboring bins. The default rectangular window results in a $13 \mathrm{~dB}$ sidelobe, which may be too big for OMP to yield accurate solutions.

Approved for public release; distribution unlimited. 


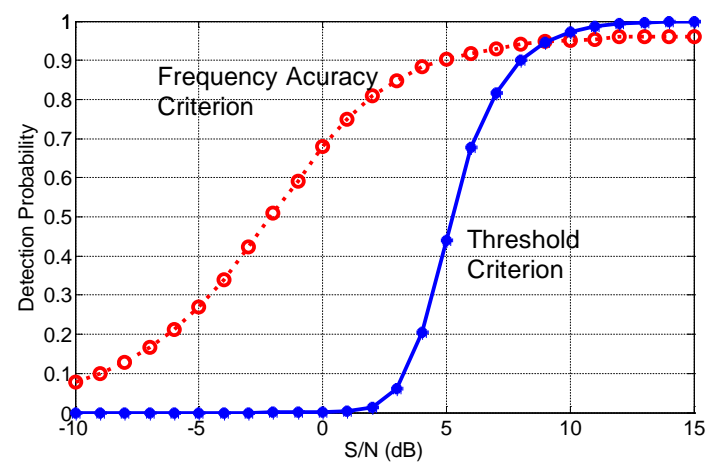

Figure 3: Detection Probability vs. S/N for PRC Case without Remedy

The measurement matrix size is 36x256. *: detection based on detection threshold; o: detection based on frequency accuracy.

A simple remedy for this problem is to apply Blackman window which is known to reduce sidelobe to $-58 \mathrm{~dB}$. Repeating the sensitivity calculation, the result after this remedy is shown in Figure 4. Here only the $\mathrm{P}_{\mathrm{d}} \mathrm{vs}$. $\mathrm{S} / \mathrm{N}$ is shown since the PDF result is similar to Figure 2 and not shown here. One notes that the gap disappears and the detection probability reach nearly $100 \%$ as $\mathrm{S} / \mathrm{N}$ increases. One also notes that the sensitivity is about $6.8 \mathrm{~dB}$ according to threshold detection (1 $\mathrm{dB}$ according to frequency-determined criterion).

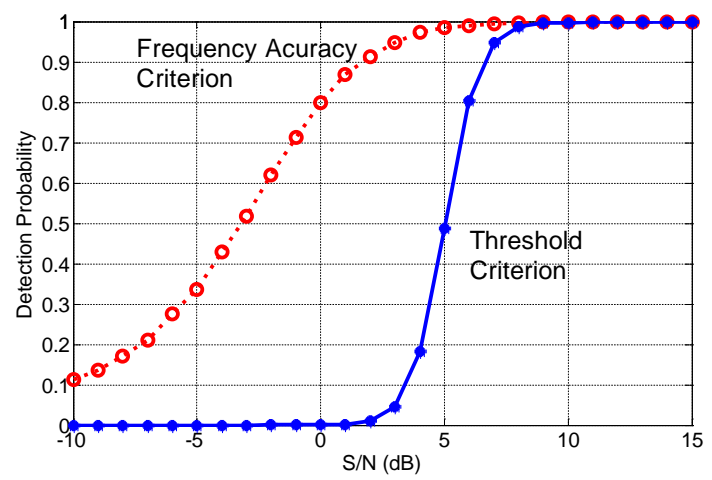

Figure 4: Detection Probability vs. S/N for PRC Case with Remedy

The measurement matrix size is 36x256. *: detection based on detection threshold; o: detection based on frequency accuracy.

Approved for public release; distribution unlimited. 


\subsubsection{NUS Results and Discussion}

Figure 5 shows the PDF of the NUS CS-based receiver using OMP. Same as the PRC case, the PDF can be approximated with a two half Gaussians and a fat tail (i.e., exponential decade) as in the following equation:

$$
\begin{aligned}
& p(x)=19141 \exp \left[\frac{-(x-0.3834)^{2}}{0.003930}\right] ; 0.3834 \geq \mathrm{x} \\
& p(x)=19141 \exp \left[\frac{-(x-0.3834)^{2}}{0.012776}\right] ; 0.6168 \geq \mathrm{x}>0.3834 \\
& p(x)=272 \exp [-26.99(x-0.6168)] ; \mathrm{x}>0.6168
\end{aligned}
$$

where $\mathrm{x}$ is the random number of the output of the NUS-based receiver using OMP when Gaussian noise is at the input. The threshold is 1.003 when false alarm rate is $10^{-7}$. Figure 6 is the detection probability vs. S/N.

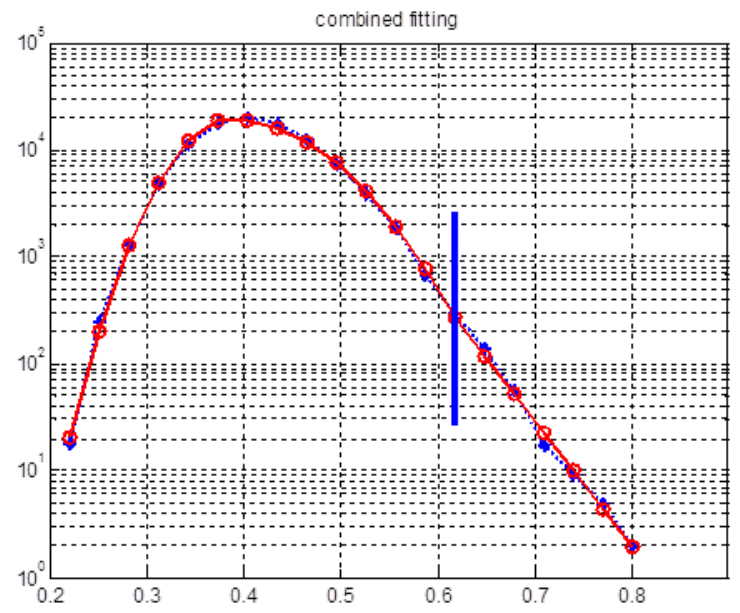

Figure 5: Probability Density Function of NUS CS Scheme Receiver

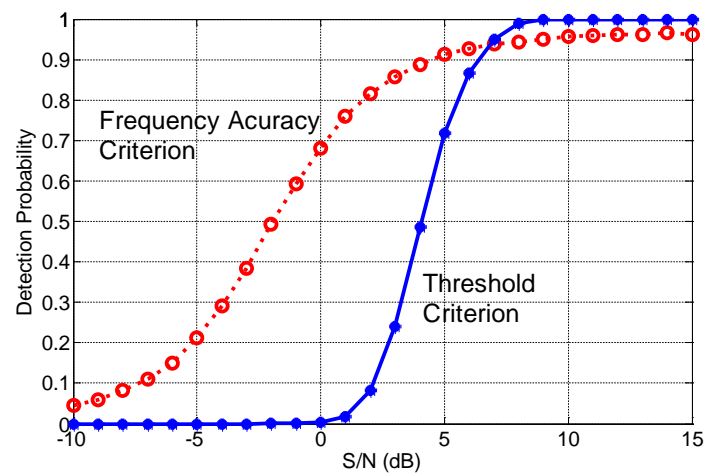

Figure 6: Detection Probability vs. S/N for NUS Case without Remedy

The measurement matrix size is 36x645. *: detection based on detection threshold; o: detection based on frequency accuracy.

Approved for public release; distribution unlimited. 
As in Figures 3 and 4, the two curves in this figure are the detection probability using either the threshold or the frequency criterion. Similar to Figure 3, the frequency-determined detection does not reach $100 \%$ when $\mathrm{S} / \mathrm{N}$ increases. First thought is to apply Blackman window as the one used for PRC case to remedy the problem. However, it does not yield improvement. The reason is due to the difference in NUS's measurement matrix which is not as robust as PRC's. Since there is only one non-zero entry for each row in NUS measurement matrix, the Blackman window only applies to that single non-zero entry, and thus exerts little effect to suppress sparcity. A close look into those cases giving wrong frequency results are shown in Figure 7. It shows that when input frequency is at the Fourier bins, the frequency detection reaches nearly $100 \%$ as $\mathrm{S} / \mathrm{N}$ increases. However, when input frequency is at the middle of two adjacent Fourier bins, the frequency detection reaches only $80 \%$ as $\mathrm{S} / \mathrm{N}$ increases. With this information, we devise a remedy for NUS CS signal detection.
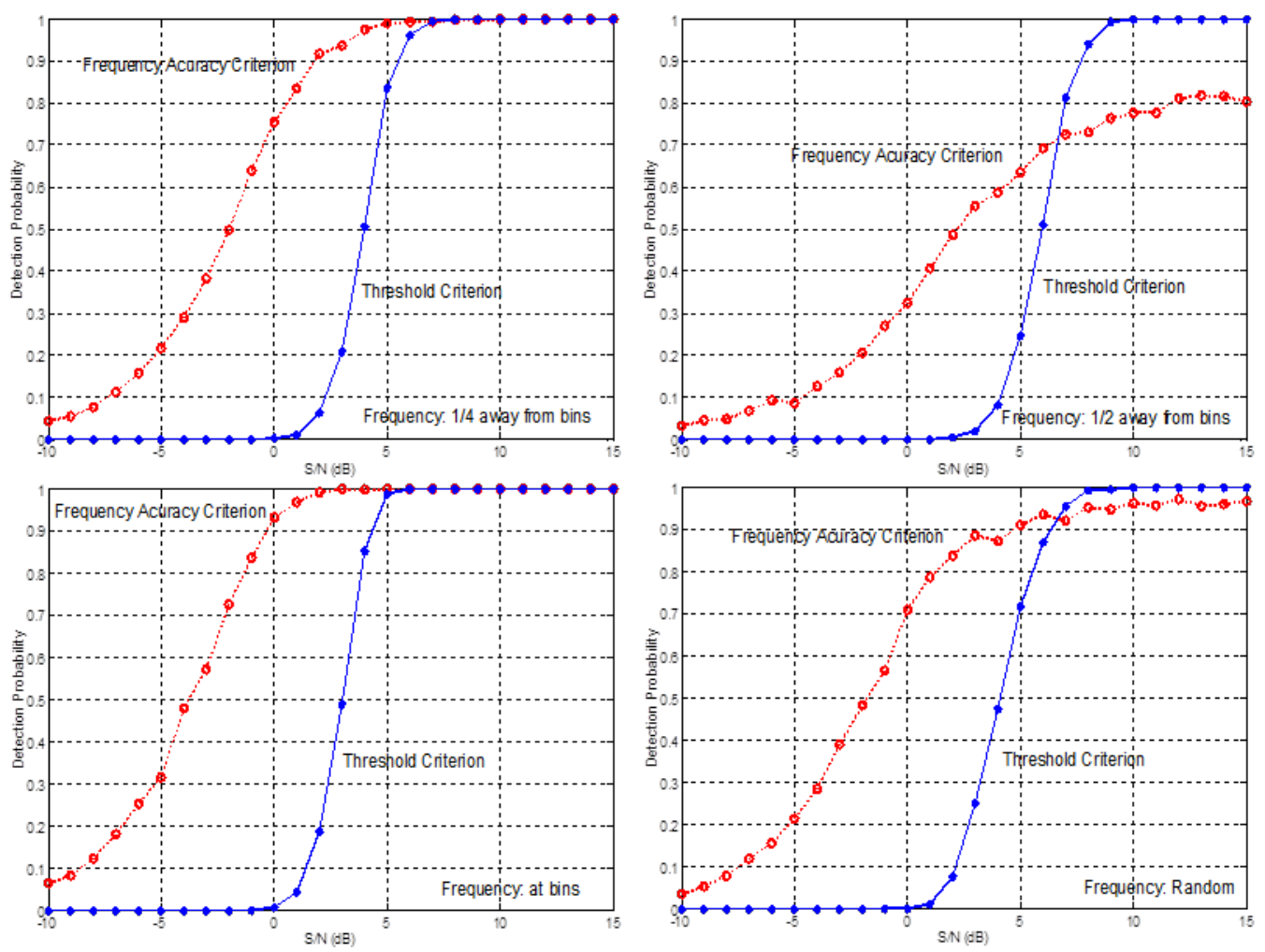

Figure 7: Probability of Detection vs. S/N when the Input Frequency is restricted to at Quarter, Half, and Zero Integer away from the Fourier Bins

The last figure has the input frequency randomized.

Approved for public release; distribution unlimited. 
In addition to the computation using the normal Fourier basis at the integer bins, we apply an extra computation using Fourier basis at half integer bins. The matrix and matrix element for the Fourier basis at the integer bins and at the half integer bins are given by:

$$
\begin{aligned}
& F_{k n}=\exp (-j 2 \pi k n / N) ; \Psi=F^{-1} \\
& F_{1 / 2, k n}=\exp (-j 2 \pi(k+1 / 2) n / N) ; \quad \Psi_{1 / 2}=F_{1 / 2}-1
\end{aligned}
$$

where $\Psi$ and $\Psi_{1 / 2}$ are the matrices for Fourier basis at integer and half integer bins, respectively. By comparing the results using $\Psi$ and $\Psi_{1 / 2}$, a more accurate frequency result can be obtained.

Repeating the sensitivity calculation, the result after this remedy is shown in Figure 8. Here only the $\mathrm{P}_{\mathrm{d}}$ Vs. $\mathrm{S} / \mathrm{N}$ is shown since the PDF result is similar to Figure 5 and not shown here. It is noted that the gap disappears and the detection probability reaches nearly $100 \%$ as $\mathrm{S} / \mathrm{N}$ increases. One also notes that the sensitivity is about $4.8 \mathrm{~dB}$ according to threshold detection $(0.8 \mathrm{~dB}$ according to frequency-determined criterion).

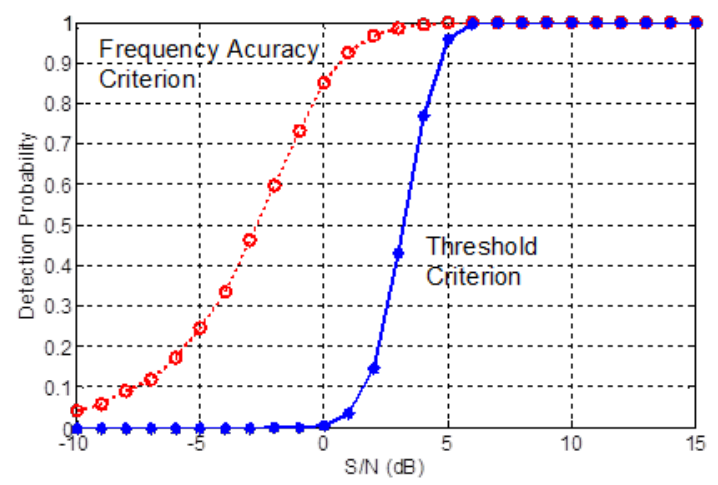

Figure 8: Detection Probability vs. S/N for NUS Case with Remedy

The measurement matrix size is 36x645. *: detection based on detection threshold; o: detection based on frequency accuracy.

Approved for public release; distribution unlimited. 


\subsection{SUMMARY AND FUTURE WORK}

Compressed sensing technology has generated interest in image and signal processing. It is of great interest to assess the performance of CS-based EW receiver in terms of sensitivity and dynamic range. We have conducted sensitivity simulation of the CS cases using PRC and NUS modulations and OMP restoration algorithm. It has been found that sparcity poises problem in frequency determination. As a result, the frequency cannot be accurately calculated even the signal is detected. For PRC case, we propose applying Blackman window in the measurement matrix to suppress sidelobe and subsequently to improve sensitivity performance of the PRC modulation case. For NUS case, we propose an algorithm that combines Fourier basis at the integer bins and at the half integer bins to improve the frequency accuracy. The results of the improvement using these remedies have been shown in this study.

The future work will continue assessing performance of the CS-based receiver using different restoration algorithms including NESTA. It will also include the hardware implementation of both measurement matrix in the front-end and signal processing in the back-end. The front-end module involves circuit design to realize PRC and NUS modulation schemes. The back-end module involves loading algorithm into computation devices such as general purpose computing on graphics processing unit (GPGPU) for signal detection and classification in real time. 


\subsection{REFERENCES}

[1] E. Candès, J. Romberg, and T. Tao, "Robust uncertainty principles: Exact signal reconstruction from highly incomplete frequency information,” IEEE Trans. Inform. Theory, vol. 52, pp. 489-509, Fenruary, 2006.

[2] M. Wakin, S. Becker, E. Nakamura, M. Grant, E. Sovero, D. Ching, J. Yoo, J. Romberg, A. E. Neyestanak, E. Candes, “A Non-Uniform Sampler for Wideband Spectrally-Sparse Environments”, IEEE Journal on Emerging and Selected Topics in Circuits and Systems, vol. 2, pp. 516-529, September, 2012.

[3] D. Takhar, J. N. Laska, M. B. Wakin, M. F. Duarte, D. Baron, S. Sarvotham, K. F. Kelly, and R. G. Baraniuk, “A New Compressive Imaging Camera Architecture using Optical-Domain Compression”, Proc: IS\& T/SPIE Symposium on Electronic Imaging: ComputJuhwan Yoo ; Turnes, C. ; Nakamura, E.B. ; Le, C.K. ; Becker, S. ; Sovero, E.A. ; Wakin, M.B. ; Grant, M.C. ; Romberg, J. ; Emami-Neyestanak, A. ; Candes, E., “A Compressed Sensing Parameter Extraction Platform for Radar Pulse Signal Acquisition”, IEEE Journal on Emerging and Selected Topics in Circuits and Systems, vol. 2, pp. 516-529, September, 2012ational Imaging, San Jose, CA, Jan. 2006, vol. 6065, pp. 43-52.

[4] G. Pope, “Compressive Sensing: A Summary of Reconstruction Algorithm, Master Thesis, Feb, 2009.

[5] S. R. Becker, "Practical Compressed Sensing: Modern Data Acquistion and Signal Processing”, PhD Thesis, California Institute of Technology, April, 2011.

[6] J. Tsui, “Special Design Topics in Digital Wideband Receivers”, 2010 Artech House, Norwood, MA.

[7] Juhwan Yoo ; Turnes, C. ; Nakamura, E.B. ; Le, C.K. ; Becker, S. ; Sovero, E.A. ; Wakin, M.B. ; Grant, M.C. ; Romberg, J. ; Emami-Neyestanak, A. ; Candes, E., "A Compressed Sensing Parameter Extraction Platform for Radar Pulse Signal Acquisition”, IEEE Journal on Emerging and Selected Topics in Circuits and Systems, vol. 2, pp. 516-529, September, 2012 


\section{LIST OF SYMBOLS, ABBREVIATIONS, AND ACRONYMS}

$\begin{array}{ll}\text { ACRONYM } & \text { DESCRIPTION } \\ \text { ADC } & \text { analog-to-digital converter } \\ \text { A-to-I } & \text { analog-to-information } \\ \text { CS } & \text { compressed sensing } \\ \text { DARPA } & \text { Defense Advanced Research Projects Agency } \\ \text { DR } & \text { dynamic range } \\ \text { ELINT } & \text { Electronic Intelligence Receiver } \\ \text { EW } & \text { electronic warfare } \\ \text { FFT } & \text { Fast Fourier transform } \\ \text { FPGA } & \text { field-programmable gate array } \\ \text { GPGPU } & \text { general purpose computing on graphics processing unit } \\ \text { NUS } & \text { non-uniform sampling } \\ \text { OMP } & \text { orthogonal matching pursuit } \\ \text { P } & \text { detection probability } \\ \text { PDF } & \text { probability density function } \\ \text { PRC } & \text { pseudorandom chip } \\ \text { RF } & \text { radio frequency } \\ \text { RIP } & \text { restrictive isometric property } \\ \text { S/N } & \text { signal-to-noise }\end{array}$

